\title{
A new product design method based on virtual reality, gaming and scenarios
}

\author{
Martijn Tideman - Mascha C. van der Voort • \\ Fred J. A. M. van Houten
}

Received: 28 July 2008 / Accepted: 11 September 2008 / Published online: 16 October 2008

(C) The Author(s) 2008. This article is published with open access at Springerlink.com

\begin{abstract}
Through the years, many methods and tools have been developed that support designers in creating good products. Current trends, for example, are to use virtual reality (VR) simulation, gaming principles, and scenario based techniques during product design processes. Each of these methods and tools contributes to the potential effectiveness and efficiency of product design processes. However, in current practice, they are often applied in an ad-hoc manner. This paper presents a new product design method that integrates elements of a number of important trends in contemporary product design processes. Using VR simulation, gaming principles and scenarios, the new product design method gives non-designers (e.g. users, production engineers, marketing managers, maintenance workers) a proactive role in the design process. Within a dedicated design environment, all stakeholders are allowed to create their own designs and immediately test these in a wide variety of use scenarios. By letting stakeholders realistically interact with their personal creations, designers can quickly and reliably pinpoint their needs and preferences. At the same time, good designs are generated. The new product design method was applied to the design of a lane change support system; a system that supports the driver of a vehicle in performing lane change maneuvers. Using the design environment that was established for this case, the designer was able to get a consistent
\end{abstract}

M. Tideman · M. C. van der Voort $(\varangle)$ · F. J. A. M. van Houten Laboratory of Design, Production and Management,

University of Twente, P.O. Box 217, 7500 AE Enschede,

The Netherlands

e-mail: m.c.vandervoort@utwente.nl

M. Tideman

e-mail: m.tideman@gmail.com

F. J. A. M. van Houten

e-mail: f.j.a.m.vanhouten@utwente.nl image of everyone's preferences as well as to draw a reliable conclusion about what would be a good design.

Keywords Product design method - Virtual reality simulation - Gaming principles $\cdot$ Scenarios $\cdot$ User centered design

\section{Introduction}

Through the years, many methods and tools have been developed that support designers in creating good products. When considering the state of the art in product design process support, a number of trends can be discerned. For example, rapid developments in computer hardware and software have made virtual reality (VR) simulation a popular design tool. Other trends in product design process support are using methods that allow simultaneous performance of different design activities, using methods that consider the product design process as a group activity, and using methods that involve users during the product design process. Also, using creativity techniques, gaming principles and scenario based techniques is becoming increasingly popular.

Each of these methods and tools contributes to the potential effectiveness and efficiency of product design processes. They all support designers in determining stakeholders preferences and finding a design that is a good compromise between those preferences. However, in current practice, they also appear to "co-exist". Elements are sometimes combined in individual product design processes, but this is only done in an ad-hoc manner. A design method that integrates elements of all those currently popular methods and tools does not exist.

This paper presents a new product design method that integrates elements of a number of important trends in 
contemporary design process support. A product design method that supports designers in determining stakeholders' preferences and finding the best compromise between those preferences. A method that allows all stakeholders to obtain insight into the consequences of decisions, that enables them to express their preferences, and that provides designers with the information necessary to draw a reliable conclusion about what would be a good design. A method that specifically supports the design of products that are new, that are complex, and that involve many different stakeholders. The new product design method is presented in Sect. 3 of this paper. Before that, Sect. 2 describes the trends on which it is based.

The new product design method was evaluated by applying it to a design case. Sect. 4 describes the design process that emerged. This process included establishing a design environment. Section 5 details the results from assessing this design process. Finally, Sect. 6 contains concluding remarks.

\section{Trends}

\subsection{Performing activities simultaneously}

The first trend that was used as a basis for the new product design method is "simultaneous performance of different activities during the product design process". This trend stems from the concurrent engineering paradigm [1]. Within this paradigm, a product design process is no longer a sequence of phases that are executed in a predefined order, as it is within a sequential engineering paradigm $[2,3]$. In fact, within this paradigm, there is no need to predefine all the phases that can occur within a product design process. All activities with regard to the design of the product - and the processes that play a role during the product's life cycle — can be performed simultaneously.

\subsection{Considering design as a group activity}

The second trend is "considering the product design process as a group activity". This trend comes from Stephen $\mathrm{Lu}$ taking the concurrent engineering paradigm one step further [4]. He sees the product design process as a group activity in which communication and collaboration plays a central role and in which the result is not only determined by technical decisions, but also by the social interaction between the various human actors involved. Differing from the long iterations of sequential engineering or the shorter iterations of concurrent engineering, his design method attempts to completely replace design iterations with negotiations. The name that $\mathrm{Lu}$ proposes for his paradigm is "engineering as collaborative negotiation" (ECN).

\subsection{Involving intended users}

The third trend is "involving intended users of the product during the product design process". This trend comes from the desire to ensure that the product functions satisfactorily in as many situations as possible. Involving users in the design process can be done in many different ways. For example, by performing market research, reasons for buying, using, possessing and discarding products are discovered. By applying Quality Function Deployment (QFD), "customer requirements" are translated into "technical requirements" [5]. Asking users to give feedback on concepts or prototype designs aims at making a correct decision about how to proceed. The largest degree of user participation is achieved by applying Participatory Design (PD). This approach prescribes that users be involved in all stages of the design process for constant evaluation of ideas, concepts and prototypes [6].

\subsection{Using scenarios}

The fourth trend originates from the discipline of software engineering: using scenarios [7]. Scenarios are explicit descriptions of hypothetical events concerning a product during a certain phase of its life cycle [8]. A scenario may be expressed by means of text, images, movies or animations. A scenario can also be expressed by displaying a prototype (either real or virtual) in an environment (either real or virtual). Within design processes, scenarios are used to address problems, needs, constraints and possibilities. This not only stimulates communication, coordination and collaboration, but also avoids misunderstandings between the involved human actors. Furthermore, because information is represented in an understandable, easily accessible, and often contextual form, using a scenario based design method allows for the inclusion of non-experts (such as intended users of the product) into the design process.

\subsection{Stimulating creativity}

The fifth trend is "applying design tools that stimulate creativity". Traditionally, within product design processes, there is an emphasis on using tools that support the representation and the analysis of design information. Today, however, it is understood that "creativity" is not a static personal characteristic, but rather one that can be stimulated. As a result, design tools that stimulate creativity - thereby supporting the generation of design information-are more frequently applied.

\subsection{Using virtual reality simulation}

The sixth trend can be attributed to rapid developments in computer hardware and software: using virtual reality (VR) simulation. A VR simulation system makes it possible for a 
human to have lifelike interaction with a computer model of a candidate design. By using VR simulation, misunderstandings between human actors are less likely to occur compared to when using more abstract or symbolic representations of design information (such as natural language, sketches and CAD drawings). Another benefit of using VR simulation is that it eliminates the necessity to make physical prototypes. It not only saves money and time, but also allows for evaluation of candidate designs in an earlier phase of the design process [9].

\subsection{Using gaming principles}

The seventh, and last, trend that was used as a basis for the new product design method is "using gaming principles". For example, Ehn and Sjögren [6] developed several different games to be used for design purposes. By playing these games, a common language was developed between designers and users. This provided the means for discussing the existing reality and for investigating future visions. From this, the requirements of the proposed product could be specified. Iacucci et al. [10] tested the principle of "design by playing games" as well. From their experiments, they conclude that playing games is a way to generate ideas in a situated and participative way. Moreover, the culture of players and the context of use of the proposed product is made explicit.

Due to rapid developments in the gaming industry, the trend to use games as a design tool no longer means just board games and card games, but also computer games. In the past couple of years, the serious game genre has emerged as a more entertaining way of revealing processes to adults [11]. Because computer games provide insight into the possible consequences of real-world decisions, they are a potentially useful product design tool. Within a computer game, information is simultaneously generated, represented and evaluated. Therefore, design iterations can be performed very quickly. Moreover, the objective of playing a game coincides with the objective of a product design process: finding a solution to a problem within constraints.

\section{The new product design method and its design environment}

\subsection{Introduction}

This section presents the new product design method that supports designers in determining stakeholders' preferences when designing products that are new, that are complex, and that involve many different stakeholders. This method incorporates the essence of the seven trends that were described in Sect. 2.
A product design method specifies activities and provides guidelines for how to perform those activities. However, it would go beyond the scope of this paper to describe those activities and guidelines in great detail. Instead, a description is given of the design process that should emerge as a result of using the new design method.

\subsection{The design environment}

The backbone of the design process is a simulation model. This simulation model consists of two elements-an environment database and a technology database. The environment database contains the set of elements that represent the world relevant to the product. The technology database contains the set of technology that might be relevant to the product (i.e. the technological potential that could be exploited by the product). Both databases are created and maintained by the designer. ${ }^{1}$ By means of a VR simulation system, stakeholders can have lifelike interaction with the contents of both databases. By means of configuration panels, stakeholders can adapt parameters of both databases, thus generating candidate designs and test environments for the candidate designs. The simulation model, the VR simulation system, and the configuration panels together form the design environment.

\subsection{The basic cycle}

A scenario is formed by a combination of elements from the environment database together with a task description. A stakeholder experiences a scenario by trying to perform the task in the simulated environment. From these experiences, the stakeholder identifies what he needs and/or wants. By combining elements from the technology database, the stakeholder can configure a candidate design with which he expects to fulfill his needs and desires.

By applying a self-configured design to a self-configured scenario, the stakeholder can assess whether his expectations about the functionality, behavior and performance of the design were correct. By applying the design to a different scenario (i.e. a different combination of elements from the environment database together with a different task description), the stakeholder can test whether it also functions satisfactorily under different circumstances or whether new needs emerge. At any point during the session, the stakeholder is allowed to alter the configuration of the design or even to start all over again with a completely different design. Similarly, at any point during the session, the

\footnotetext{
${ }^{1}$ Unless explicitly indicated, the word "designer" may also be read as "design team".
} 
Fig. 1 The higher level processes during application of the new product design method

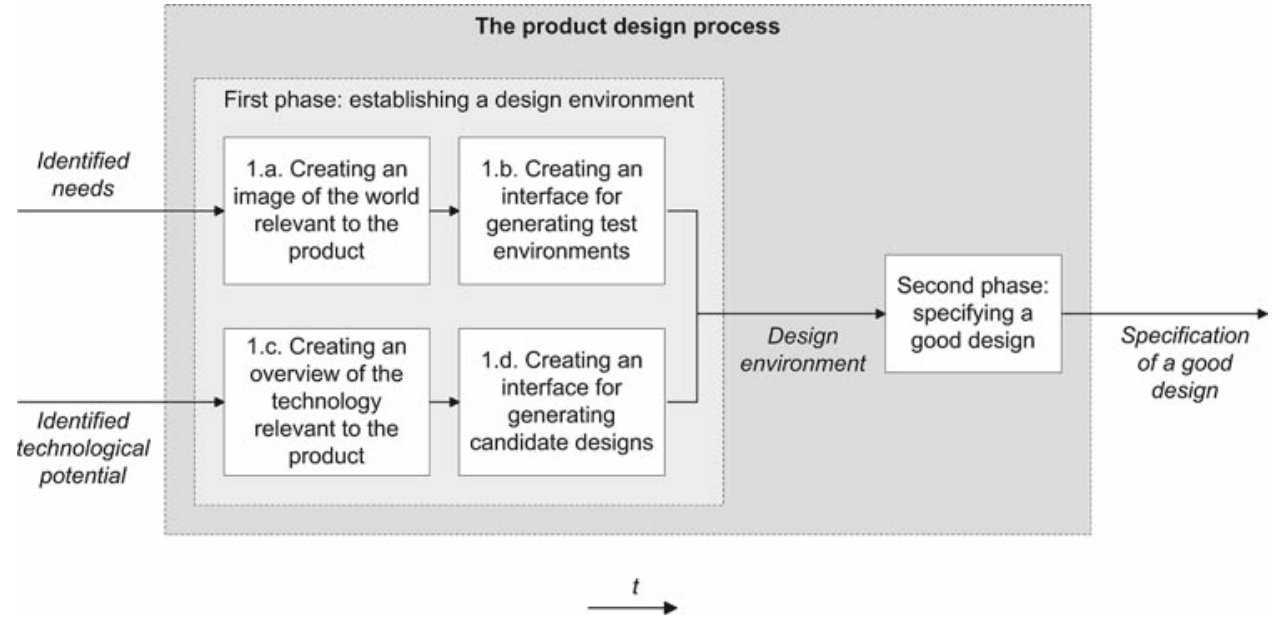

stakeholder is allowed to alter the configuration of the scenario or even to start all over again with a completely different scenario.

\subsection{Two phases}

The design process is split into two separate phases. The first phase is aimed at developing the design environment into a valid representation of the world relevant to the product and the technology that may be usefully applied to the product. During the second phase, the design environment-as it was created during the first phase-is used to specify a good design.

Figure 1 shows the higher level processes during application of the new product design method. It shows that, while applying the new method, identified needs and identified technological potential are converted into a specification of a good design:

- From identified needs, an image of the world relevant to the product is created (process 1.a). This image consists of an environment database and a VR simulation system to have lifelike interaction with the contents of the database. Also, an interface is created with which stakeholders can generate test environments by selecting combining, and adapting environment parameters (process 1.b);

- Identified technological potential is used to create an overview of the technology relevant to the product (process 1.c). This image consists of a technology database and a VR simulation system to have lifelike interaction with the contents of the database. Also, an interface is created with which stakeholders can generate candidate designs by selecting combining, and adapting technology parameters (process 1.d);

- The image, the overview, and both interfaces together form the design environment;
- During the second phase of the design process, the design environment is used to specify a good design.

Sections 3.5 and 3.6 give a more detailed description of the procedure during the respective phases of the design process.

\subsection{Procedure during the first phase}

The first phase starts with activities such as observing the real-world, reading literature and talking to stakeholders. Based on the results of such activities, the designer makes an initial assumption about the necessary contents of both the environment database and the technology database. In other words, the designer attempts to identify all aspects of the world relevant to the product as well as all technology that may be usefully applied to the product. Based on the results from this identification process, the designer creates an initial simulation model. Simultaneously, the designer creates a VR simulation system that enables stakeholders to have lifelike interaction with the simulation model, as well as configuration panels that give stakeholders the possibility to generate candidate designs and test environments.

After the initial design environment has been established, its validity is tested. More specifically, the designer tests whether all relevant aspects of the design case are present and whether they are correctly modeled. This is done by inviting stakeholders for reflection sessions. During a reflection session, a stakeholder is told that the goal is to create the most satisfying "personal design" of the proposed product. By generating designs and scenarios, and evaluating those designs in the scenarios, the stakeholder is able to iteratively work towards this goal. In the meantime, the designer observes the stakeholder's behaviour and asks the stakeholder for opinions about the generated designs. However, the designer only performs these activities in order not to reveal the true purpose of the session. This true purpose (i.e. collecting feedback on the quality of the design environment) is 
only accomplished at the end of the reflection session when the stakeholder is interviewed. The feedback from all stakeholders is used to create a list of required adaptations to the design environment.

The designer implements the required adaptations into the design environment. Subsequently, the same people that participated in the reflection sessions are invited again, but now for verification sessions. During a verification session, a stakeholder is confronted with the adaptations to the design environment that he explicitly or implicitly proposed. The stakeholder is asked whether he agrees to the adaptation or whether something else was intended. Additionally, the stakeholder is confronted with adaptations that were proposed by others. This time, the stakeholder is not asked whether he agrees to this adaptation. Instead, the stakeholder is asked whether he rejects the adaptation; not rejecting an adaptation is considered sufficient for acceptance of the specific adaptation.

By performing this cycle of reflection sessions, implementation of the required adaptations and verification sessions, the design environment evolves. Initially, the design environment will evolve rapidly. After a certain number of cycles, the speed of evolution will decrease. Ultimately, the evolution of the design environment will practically stop-stakeholders will only be able to confirm the completeness and correctness of the design environment. The design environment has become "saturated". It will contain the "problem-solution space" of the design case in a form that is both verifiable and controllable. When this state of saturation is achieved, the first phase of the new product design method has come to an end.

\subsection{Procedure during the second phase}

During the second phase of the design process, the design environment - as established during the first phase-no longer changes. There is now an emphasis on the quality of the proposed product, instead of on the quality of the design environment. Stakeholders are invited for design sessions. The activities of the stakeholders during these design sessions are quite similar to the activities during the reflection sessions of the first phase of the design process. A stakeholder is given the assignment to iteratively work towards the "most attractive design" by generating designs and scenarios, and evaluating those designs in the scenarios. In the meantime, the designer observes the stakeholder's behavior and asks the stakeholder for opinions about the generated designs. In contrast to during the first phase of the design process, the designer now actually uses the collected information. For every stakeholder, the designer creates a "personal report". This personal report contains both objective information (i.e. personal information and a specification of the "most attractive design") and subjective information (i.e. reasons for why the specified design is so attractive and why other product features are less desirable). Based on these personal reports, the designer specifies (an) attractive design(s) for each stakeholder.

Because of the large amount of parameters that stakeholders could adapt to generate candidate designs, it is unlikely that all "attractive designs" are identical. It is therefore likely that the designer has to search for a compromise between the preferences of all stakeholders. All information from the personal reports (both the objective and the subjective information) is organized into a hierarchy. Such a hierarchy enables the designer to specify the "best" design within any set of constraints, for example, constraints set by other stakeholders.

\section{Application of the new product design method}

\subsection{Introduction}

To learn how the new product design method performs in practice, it was applied to a design case. During performance of this design case, two processes ran in parallel: the design process and the assessment process. The design process is the process of applying the new product design method to the case. The assessment process is the process of collecting and analyzing data about the design process that emerged as a result of applying the new product design method to the case. The design process is presented in this section. Section 5 describes the assessment process.

\subsection{Selected design case}

A lane change support system was selected as a design case. This is a product that supports drivers of a vehicle in performing lane change maneuvers. It is a new product (no lane change support system was on the market when the design case was started in January 2005). It is a complex product (a lane change support system has many interrelated design parameters, it can be implemented in many different ways, and it is used in a complex environment). It is also a product that involves many different stakeholders who may all have different needs and desires (e.g. OEMs, suppliers, governments, legal institutions, and-last but not least-car drivers). In short, it is a typical example of a product for which the new product design method was developed.

\subsection{Simplifications}

Because of a limited availability of resources, a number of simplifications were implemented. Firstly, the design case was performed by a single designer rather than by a design team. Secondly, an interface for stakeholders to generate test 
environments was not created. Instead, the designer generated the scenarios that were offered to stakeholders to evaluate their self-configured designs. Thirdly, the design process was only performed for the use aspects of a lane change support system. Other aspects (such as production, marketing, maintenance, etc.) were not represented by the design environment. As a result, only representatives from the stakeholder group "users" were involved in the design process.

\subsection{The first phase of the design process}

\subsubsection{Creating the initial design environment}

Results from observing the real-world, talking with stakeholders, and performing a literature study (see, for example [12]) were converted into an initial design environment. It consisted of the following main elements:

- An environment database filled with traffic scenarios.

- A technology database filled with lane change support system technology.

- A lane change support system configurator that enabled a user to generate lane change support system designs.

- A driving simulator that enabled a user to control a vehicle within the traffic scenarios, thereby realistically experiencing them. The driving simulator also allowed the user to realistically experience the behavior of the lane change support system designs within the traffic scenarios.

Figure 2 shows the initial design environment. It was made up of a mock-up of a vehicle, a large curved screen that displays the traffic environment, and a sound- system that displays sounds from the traffic environment. The mock-up itself consisted of a force feedback steering wheel and a pedal set, a driver's seat equipped with vibrating elements, four flat screens that together form a dashboard, three flat screens that offer rear view mirror functionality, and an in-car sound sys-

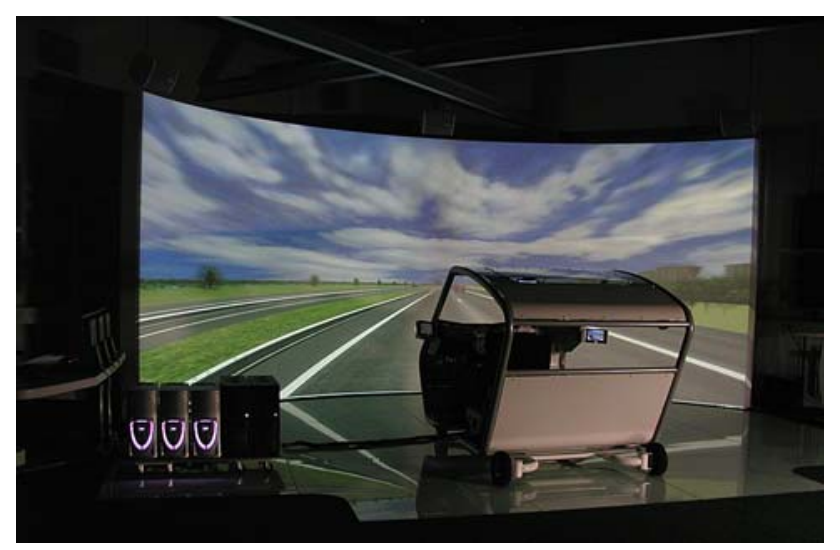

Fig. 2 The initial design environment

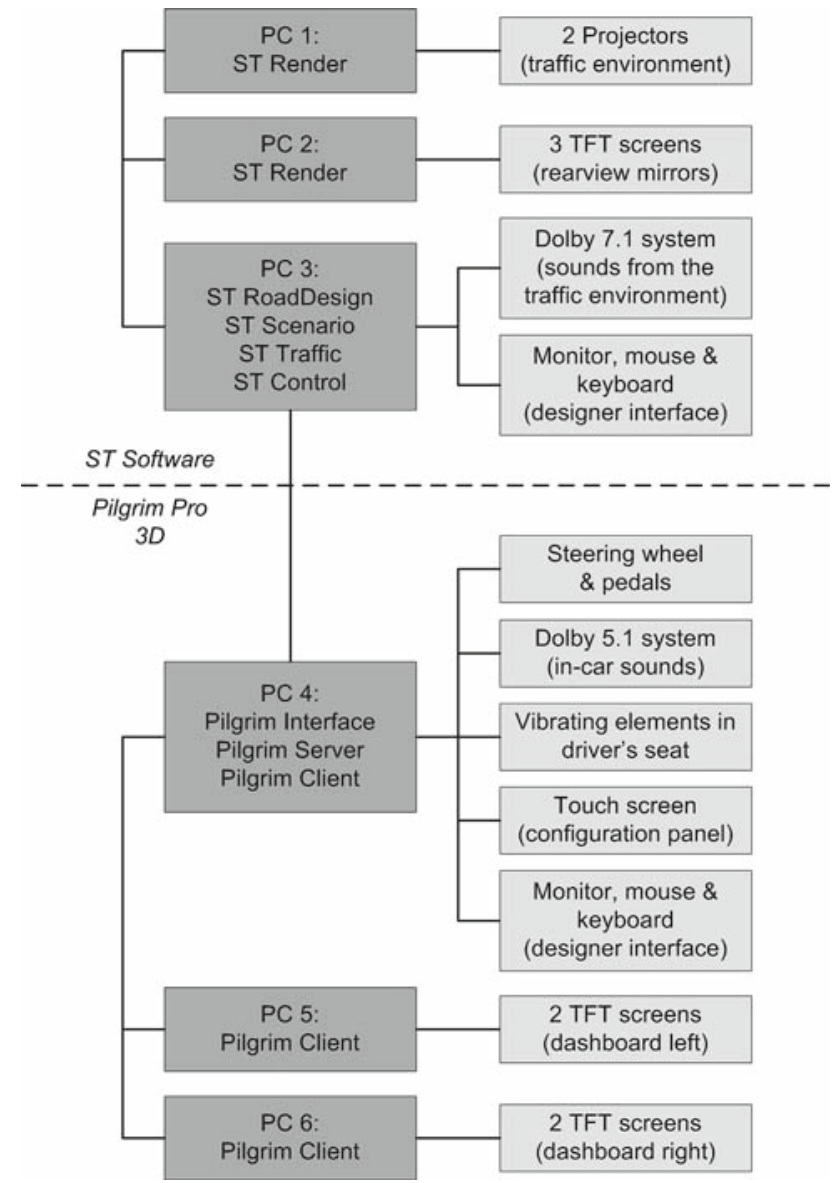

Fig. 3 The design environment's hardware architecture

tem. In the middle console, a touch screen was integrated as an interface for the lane change support system configurator.

Figure 3 depicts the design environment's hardware architecture. It can be seen that the hardware interfaces in the design environment were connected to a network of six highend PCs that run on Windows XP. The simulations were controlled by two software packages: ST Software (www. stsoftware.nl) and Pilgrim Pro 3D (www.pilgrim-visuals. com). ST software is dedicated driving simulator software. Pilgrim Pro 3D is a generic 3D engine. Each software package "owned" three PCs in the network. The two software packages communicated with each other through an UDP channel.

ST Software consists of a number of modules that, together, provide driving simulator functionality.

The ST RoadDesign module is used to create road networks. It generates both graphical networks for the human driver and logical networks for the traffic participants that are controlled by the ST Traffic module. The graphical networks are built in the OpenSceneGraph (OSG) format, an open source 3D graphics toolkit. Using ST RoadDesign, the designer makes a 2D design of a road network which is then 
automatically converted into a 3D world. Within the design environment, ST RoadDesign is installed on PC 3.

The ST Scenario module is a scripting tool that generates scenarios. In this context, a scenario is a predefined list of situations with a start and an end condition. Each scenario activates and terminates automatically when required. Any number of scenarios can be active at the same time. Among other things, scenarios generate traffic participants, define the starting point of the vehicle in the road network, and provide navigation instructions to the driver. ST Scenario uses the third-party text editor TextPad for creating script files and performing syntax checks on the scripts. Within the design environment, ST Scenario is installed on PC 3.

The ST Traffic module provides real-time simulations based on the ST Scenario scripts and the logical network that was created by ST RoadDesign. Within the design environment, ST Traffic is installed on PC 3.

The ST Control module is the graphical interface for the designer. Using ST Control, the designer can configure all ST Software modules and control the communication between those modules. ST Control is also used to start and stop traffic scenarios. Within the design environment, ST Control is installed on PC 3.

The ST Render module is a real-time renderer that enables real-time visualization of the simulated traffic environment. ST Render receives real-time simulated traffic data from ST Traffic and displays it onto one or more connected graphical displays. In ST Render, viewing angles, viewing positions and viewports can be defined. Within the design environment, ST Render is installed on PC 1 and on PC 2.

Just like ST Software, Pilgrim Pro 3D also consists of multiple modules. Together, these modules allow a user to design a lane change support system and experience this design in the traffic environment that is provided by ST software.

The Pilgrim Interface module is a tool that is used to write Lua script files. When loaded into the Pilgrim Server module, these script files form the lane change support system configurator. Each question in the electronic questionnaire has its own script file. A script file loads a question, lists the possible options, and allows a user to choose one of those options. A script file also communicates the design choice to the Pilgrim Server module. Finally, a script file directs the user to the next question to be answered. This next question may depend on an answer given earlier in the questionnaire. Within the design environment, Pilgrim Interface is installed on PC 4.

The Pilgrim Server module is the core of the Pilgrim network. This is where the lane change support system design is generated and simulated in real-time. Using the $\mathrm{C}++$ Software Development Kit (SDK), the designer fills the technology database with assistants. Assistants can be visual, auditory, tactile or kinesthetic. Every assistant has a set of attributes. Examples of attributes are "brightness", "size", "pitch", "volume", "repetition frequency", "amplitude" and "interface ID". Every assistant also has a set of conditions under which it will become active. Based on the design choice of the user received from the Pilgrim Interface module, an assistant may be created, values of attributes may be set, or conditions under which the assistant becomes active may be defined. Within the design environment, Pilgrim Server is installed on PC 4.

The Pilgrim Client module is a real-time renderer. When Pilgrim Server commands that a specific assistant should become active, Pilgrim Client will render it. Depending on the specific modality of the assistant, this rendering might be done on a visual, auditory, tactile or kinesthetic display. Within the design environment, Pilgrim Client is installed on PC 4, PC 5 and PC 6.

\subsubsection{Verifying the design environment}

Twelve users were invited for reflection sessions. A reflection session involved one user at a time. The real purpose of the reflection session (i.e. checking the design environment for completeness and correctness) was not revealed. Rather, users were told that the session was aimed at finding out which lane change support system design is most desirable. After having become familiar with the design environment, users were offered the opportunity to iteratively work towards their personal "most attractive design". They did this by generating lane change support system designs and by evaluating them in the scenarios that were offered by the designer. Figure 4 shows a user generating a candidate design. Figure 5 shows a user evaluating a candidate design.

At the end of a reflection session, the user was asked for feedback about the quality of the design environment. More specifically, the user was asked whether all elements relevant to a lane change support system were present in the design

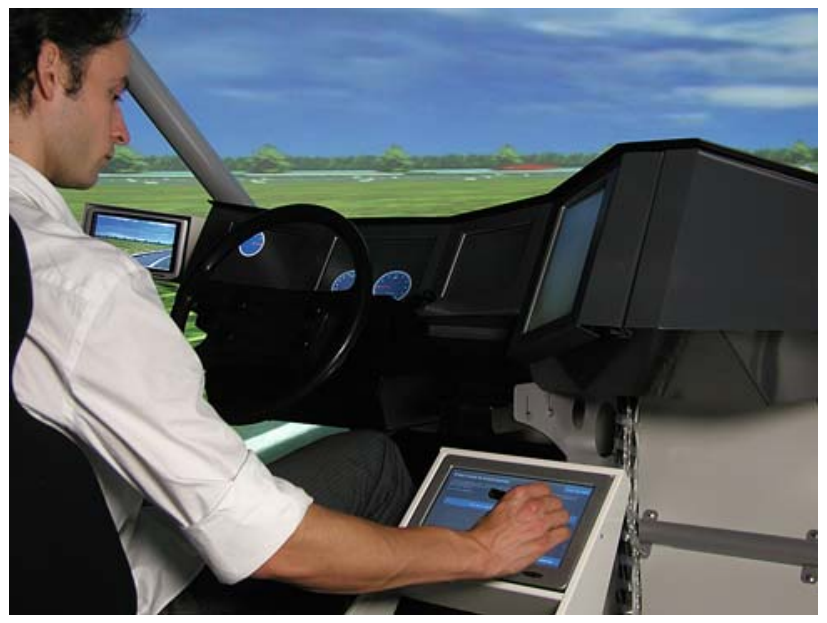

Fig. 4 Generating a candidate design 


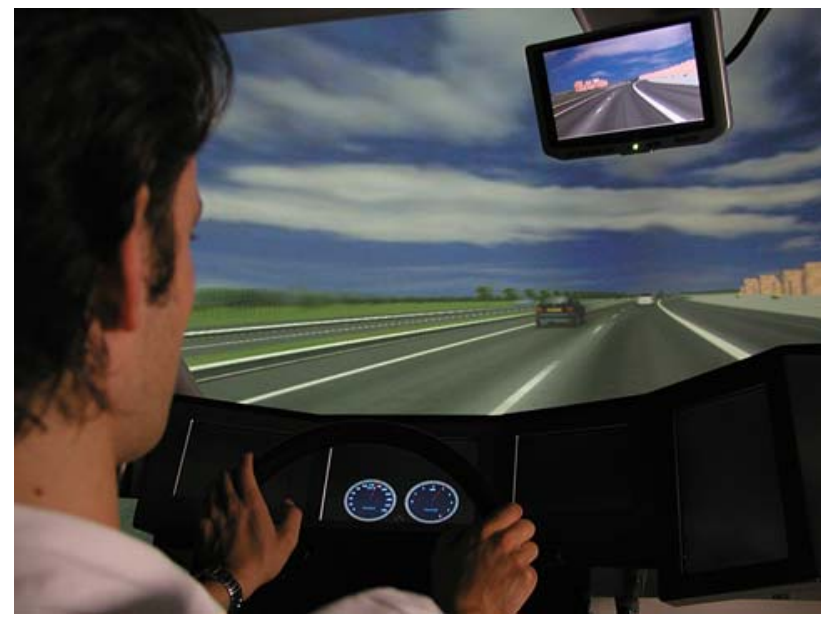

Fig. 5 Evaluating a candidate design

environment, and whether all present elements were correctly modeled. From the feedback that was collected from all users followed that a total of 30 adaptations needed to be implemented: 17 to the environment database, 8 to the technology database and 5 to the lane change support system configurator. An important finding was that a lane change support system should not be considered as one integrated system, but rather as an assembly of independently functioningand independently configurable-assistants (i.e. modules). For every assistant, users should be able to specify the "circumstances under which support is provided", the "contents of the provided support", and the "form of the provided support". The working principle of the support system as a whole is simply the sum of the working principles of the assistants.

After having implemented the adaptations, the same group of users was invited again, but now for verification sessions. The objective of the verification sessions was to test whether the quality of the design environment had improved. It appeared that all users confirmed that the personally proposed adaptations had been implemented correctly (i.e. implemented as they had intended them). Generally, users also agreed that the adaptations proposed by others improved the quality of the design environment. Incidentally, users proposed new adaptations during the verification sessions.

By performing one iteration of the first phase of the design process (i.e. one cycle of reflection and verification sessions), the design environment had become a better representation of the problem-solution space of a lane change support system. Performing another iteration (i.e. a new cycle of reflection and verification sessions with a new group of users) would have undoubtedly resulted in an even better representation. However, because the design process was not performed to develop the most promising lane change support system, but to evaluate the new product design method, the first phase of the design process was concluded and the second phase was started.

\subsection{The second phase of the design process}

Forty-eight users were invited for design sessions in which they iteratively had to work towards their personal "most attractive design". A design session involved one user at a time. The behavior of users while generating and testing candidate designs, and the opinions of users while reflecting on them, was registered by audio/video recordings and by notes. In addition, the interactions with the lane change support system configurator (i.e. the design choices of users) were also automatically stored in data files. For every user, the raw data collected during the design sessions were converted into a "personal report". Each personal report contained four sections:

1. Personal information of the user (age, driving style, gender).

2. A complete specification of the lane change support system design that was marked "most attractive" by the user.

3. An explanation for why the specified system was so attractive for the user. This explanation also detailed system features that were not present in the "most attractive system", such as reasons for why certain system features are considered undesirable.

4. A brief description of the user's behavior during the design sessions with a special attention to striking incidents, actions and behaviors.

The specifications of the "most attractive system" in all personal reports were compared with each other. It appeared that none of the specifications were identical. In other words, 48 different users had created 48 different "most attractive systems". Sometimes, differences between users' specifications could be characterized as "detailed differences". However, within the total set of 48 personal reports, there were many "fundamental differences": system features that were considered "fantastic" by the one user were considered "horrible" by the other user (and vice versa). This was, for example, the case for tactile support, kinesthetic support and the continuous issuance of beeps to indicate the presence of other vehicles. It was therefore impossible to specify one single lane change support system design that is attractive to all users.

In order to search for a compromise between the preferences of all users, all design information (both the objective and the subjective information) was organized into a hierarchy that is meaningful from a user's perspective. This was done by following an approach similar to "grounded theory" [13]. Originally, the aim of grounded theory is to develop a theory that fits a set of collected data. However, it has also 
become increasingly popular in product design processes to answer specific questions and address design concerns [14]. Within grounded theory, identification of themes, and organizing these into a hierarchy, is achieved by "coding" the data.

A random sample of personal reports was used to make an assumption about the set of codes from which the coding system should be made up. For every statement in these personal reports, one or more codes were defined. The defined codes were combined into a list. A trained rater was asked to assign one or more codes from the list to every statement in the sample of personal reports. Next, the average inter-rater reliability per statement (the percentage of the assigned keywords per statement that matches between the designer and the rater) was calculated. The reliability was $75 \%$, which was considered sufficient to accept the coding system as a definition of relevant themes. The three main categories of this coding system were:

- Desirable/undesirable system features.

- Reasons for considering a system feature to be desirable/undesirable.

- Circumstances under which a system feature is considered desirable/undesirable.

The coding system was used to code the subjective information in all personal reports and re-organize the objective information into categories that are meaningful from a user's perspective. Because the coding system has a hierarchical structure, this resulted in a hierarchy of information that represents the preferences of all users. A hierarchy of which the structure is meaningful from a user's perspective and that enables the designer to specify the "best" design within any set of constraints.

To illustrate this, a constraint from a lane change support system manufacturer was imposed: A lane change support system should be modular. Every assistant (i.e. every module) should function independently of the other assistants (i.e. the other modules) in the system. Users may or may not order specific assistants to be installed in their vehicle. A total of six assistants should be offered: two "comfort assistants" (one for every side of the vehicle) that give feedback about the traffic environment, independent of the intention to change lanes, two "safety assistants" (one for every side of the vehicle) that issue a warning when the user makes a mistake (i.e. when the user has the intention to change lanes whereas another vehicle is present in an adjacent zone), and two "safety assistants" (one for every side of the vehicle) that impose an intervention when the user makes a mistake.

By combining this constraint with the preferences of users as reflected by the hierarchy of information, the specifications of the six assistants were deduced. The hierarchy of information was also successfully used to specify why users would be attracted to every assistant, what they might not like about them, and how this is related to the circumstances under which they use them.

\section{Assessment of the new product design method and its design environment}

\subsection{Approach}

To evaluate whether the new product design method supports designers in determining stakeholders' preferences and finding the best compromise between those preferences, it was assessed whether the method is viable and whether it fulfills its functions. The new product design method should be viable in the sense that human actors (i.e. the designer and the stakeholders) should understand their role in the design process, be able to perform the specified activities, and that these activities should yield actual results. The new product design method should fulfill its functions in the sense that it should stimulate and enable the designer to create a consistent image of everybody's preferences and to specify a reliable compromise between all those preferences. By allowing stakeholders to create their own designs and immediately test those in a wide variety of scenarios, the created design environment should support these functions and activities.

\subsection{Method}

Hypotheses about the viability of the new product design method and about the degree to which it fulfills its functions were formulated. For every hypothesis, criteria for acceptance were specified. To test the hypotheses, three different types of data were collected during the design process. Firstly, after every activity, the designer and the users had to fill out a questionnaire in which they were asked whether they understood what was expected from them, and whether they possessed the abilities to meet those expectations. The questionnaires were also used to ask whether they felt stimulated and enabled to perform the specified activities. Secondly, direct observations as well as indirect observations (i.e. audio/ video recordings) of the designer and the users were made. These observations were used to check for consistency between the users' and designer's behavior and their answers on the questionnaire. Thirdly, the design information that was generated during the design process (e.g. notes, documents, the design environment) was collected. This data type was mainly used to check whether the specified activities yielded actual results.

\subsection{Results}

From testing the hypotheses about the viability of the new product design method appeared that the designer and all 
users understood their role in the design process and were able to perform all required activities. It also appeared that all activities yielded actual results. The design information that was generated during every activity was always in line with one of the possible outcomes as specified by the guidelines. This led to the conclusion that the new product design method is viable.

From testing the hypothesis about the degree to which the new product design method fulfills its functions appeared that people were sufficiently stimulated and enabled to generate design information, to represent the generated design information such that it is easily accessible, and to make sure that the represented design information is consistent. It also appeared that people were sufficiently stimulated and enabled to give opinions about the represented design information, to verify their opinions about the represented design information, to weigh positive and negative consequences of choices, and to find compromises.

By allowing participants to create their own designs and immediately test those in a wide variety of scenarios, the design environment supported these functions. Since the design's behavior could be actually experienced (rather than only imagined), the design environment provided clear insight into the impacts of a specific design under all kinds of different use circumstances.

All this resulted in a consistent image of each user's preferences as well as in a reliable compromise between all those preferences. It was therefore concluded that the new product design method fulfills its functions.

\section{Conclusion}

This paper presented a new product design method that supports designers in determining stakeholders' preferences and finding the best compromise between those preferences. It is based on seven trends in contemporary design process support. The new method involves establishing a dedicated design environment in which VR simulation, gaming principles and scenarios are combined. This design environment enables non-designers (e.g. users, production engineers, marketing managers, maintenance workers) to have a proactive role in the design process. Within the design environment, all stakeholders are allowed to create their own designs and immediately test these in a wide variety of use scenarios. By letting stakeholders realistically interact with their personal creations, designers can quickly and reliably pinpoint their needs and preferences. At the same time, good designs are generated.

The new product design method was evaluated by applying it to a design case: the design of a lane change support system. Data about the design process that emerged were collected and analyzed. It was found that the new product design method is viable in the sense that people understood their role in the design process, were able to perform the specified activities, and that these activities yielded actual results. It was also found that the new product design method fulfils its functions in the sense that it stimulates and enables the designer to create a consistent image of everybody's preferences and to specify a reliable compromise between all those preferences.

Although the new product design method was assessed within the confines of a specific design case, it is expected that the method can be successfully applied to the design of all products that have a certain level of modularity or configurability. It should, however, be technically possible to establish a design environment such that stakeholders can make a reliable assessment of the designs' properties; it should be technically possible to create interfaces for stakeholders to generate candidate designs and test environments, and to offer simulations of those designs in those environments. Currently, there is only a limited number of products that comply to this prerequisite. On the other hand, as VR simulation technology and information visualization techniques mature, the number of products to which the new product design method can be successfully applied will increase.

Although the new product design method is expected to be applicable to any product that has a certain level of modularity or configurability, the added value of applying it will not always be worth the investment; creating a dedicated design environment, performing sessions with stakeholders, and continuously making sure that all information is consistent, costs a significant amount of time and money. In general terms can be said that the newer the product, the more complex the product, and the more different stakeholders are involved, the higher the chances of "return on investment".

The investment will certainly be returned when the new product design method is used to design products that should be "first time right". Products such as, for example, a lane change support system. If faulty versions of such products are released, they could endanger users and seriously damage the manufacturer's position in the market.

Open Access This article is distributed under the terms of the Creative Commons Attribution Noncommercial License which permits any noncommercial use, distribution, and reproduction in any medium, provided the original author(s) and source are credited.

\section{References}

1. Sohlenius, G.: Concurrent engineering. Ann. CIRP 41(2), 645-655 (1992)

2. Pahl, G., Beitz, W.: Engineering Design: a Systematic Approach. Springer, Berlin (1984)

3. Suh, N.P.: The Principals of Design. Oxford University Press, New York, USA (1990) 
4. Lu, S.C.Y.: Engineering as collaborative negotiation: a new paradigm for collaborative engineering research, Whitepaper. http:// wisdom.usc.edu/ecn (2003). Retrieved 4 May 2006

5. Akao, Y.: Quality Function Deployment. Productivity Press, New York, USA (1990)

6. Ehn, P., Sjögren, D.: From system descriptions to scripts for action, In: Greenbaum, J., Kyng, M. (eds.) Design at Work: Cooperative Design of Computer Systems. Lawrence Erlbaum, Hillsdale, USA (1991)

7. Carroll, J.M.: Five reasons for scenario based design. Interact. Comput. 13(1), 43-60 (2000)

8. Miedema, J., van der Voort, M.C., Lutters, D., van Houten, F.J.A.M.: Synergy of technical specifications, functional specifications and scenarios in requirement specification. In: Proceedings of the 17th International CIRP Design Seminar, Berlin, Germany (2007)

9. Tideman, M., van der Voort, M.C., van Houten, F.J.A.M.: Haptic virtual prototyping for design and assessment of gearshifts. In: ElMaraghy, H.A., ElMaraghy, W.H. (eds.) Advances in Design, pp. 461-472. Springer, Berlin (2006)
10. Iacucci, G., Kuutti, K., Ranta, M.: On the move with a magic thing: role playing in concept design of mobile services and devices. In: Proceedings of the 3rd Conference on Designing Interactive Systems, pp. 193-202 (2000)

11. Bakie, R.T.: A brief history of video games. In: Rabin S. (ed.), Introduction to Game Development. Charles River Media, Hingham, USA (2005)

12. Tideman, M., van der Voort, M.C., van Arem, B., Tillema, F.: A review of lateral driver support systems. In: Proceedings of the 10th International IEEE Conference on Intelligent Transportation Systems, Seattle, USA (2007)

13. Sharp, H., Rogers, Y., Preece, J.: Interaction Design: Beyond Human-Computer Interaction, 2nd edn. Wiley, Chichester, UK (2007)

14. Glaser, B.G., Strauss, A.L.: The discovery of grounded theory: strategies for qualitative research. Aldine, London, UK (1967) 